\title{
The Measurement Situation of Concentrations of Gaseous Hydrocarbons
}

\author{
Minxuan Li \\ Mechanical and Material Department, North China University of Technology, Beijing, China \\ Email address: \\ 1013914785@qq.com
}

To cite this article:

Minxuan Li. The Measurement Situation of Concentrations of Gaseous Hydrocarbons. International Journal of Environmental Monitoring and Analysis. Vol. 5, No. 5, 2017, pp. 137-142. doi: 10.11648/j.ijema.20170505.14

Received: August 11, 2017; Accepted: September 27, 2017; Published: October 27, 2017

\begin{abstract}
Nowadays, there is an increasing number of vehicles on the road, and because of that, the air pollution is more serious than before. Although there is a three-way Catalyst Converter in every car, which can transform the $\mathrm{CO}, \mathrm{HC}$ and NOX to the harmless ingredients like $\mathrm{H}_{2} \mathrm{O}$ from the exhausted fumes. It is not sure that all the gas, such as: $\mathrm{HC}$ can be transform entirely. Thus, many vehicle manufactures intend to find some equipment to control and measure the concentration of hydrocarbons from exhausted fumes to meet the requirement of vehicle emission of world standard. This report may help the vehicle companies to solve this problem. This report first mainly describes the specific chemical types of the gaseous hydrocarbon in exhausted fume which are Alkanes, Alkenes, Alcohols, and Ether. And it also posts the disadvantages of these toxic chemicals in exhausted fumes, for instance, it is harmful to human and it pollutes the environment. Secondly, the report introduces some approaches to measure the concentration of hydrocarbon in exhausted fume from vehicles, for examples, Mass Spectrometry and Gas Chromatography, The flame ionization detector, Infrared spectroscopy, and Raman Spectrum. Furthermore, these approaches are used as a medium to find the companies who can provide such equipment to test the concentration of hydrocarbon: Horiba, AVL, and Ocean Optics. The third, the product data from these three companies is listed and compared with each other. Finally, the evaluation result of instruments to measure the concentration of gaseous hydrocarbons is shown as an input to vehicle manufactures.
\end{abstract}

Keywords: Concentration of Gaseous Hydrocarbons, Approaches, Data of Products

\section{Introduction}

It is commonly believed that exhausted fume from gasoline with lead is extremely harmful to human nature, thus there is a new standard to appeal people to use leadless gasoline in 1970s [1]. The substitution for the lead are alcohols and ethers. The gasoline with alcohols, ethers and other oxide can rise the octane number which means that the gasoline can enhance the ability of anti-vibration, meanwhile this type of gasoline can also drop the volatility. The concentration of the exhausted fume and some volatilize gas from vehicles are in relation to the oxide of fuels. The main purpose of this paper is to measure the concentration of gaseous hydrocarbons.

In organic chemistry, hydrocarbons are the compound consisting of element hydrogen and carbon. The hydrocarbons are mainly from the crude oil which is non-renewable energy on Earth. The organic matter in crude oil are enable to provide a great number of carbon and hydrogen which can catenate to form infinite chains when they are bonded.

The hydrocarbons can be divided into three groups. The first is saturated hydrocarbons which are the simplest type. They are single bonds and are saturated with hydrogen, for example, the alkanes whose formula is $\mathrm{C}_{n} \mathrm{H}_{2 n+2}$. However, the common form of the saturated hydrocarbons are $\mathrm{C}_{\mathrm{n}} \mathrm{H}_{2 \mathrm{n}+2(1-\mathrm{r})}$, where $r$ is the number of rings. This type of hydrocarbons are linear or branched species which also constitute the basis of petroleum fuels. They are founded in structural isomers. The second is unsaturated hydrocarbons which have one or more bonds between carbon atoms. For instance, alkenes has double bonds and the formula is $\mathrm{C}_{\mathrm{n}} \mathrm{H}_{2 \mathrm{n}}$, while alkynes has triple bonds and the formula is $\mathrm{C}_{\mathrm{n}} \mathrm{H}_{2 \mathrm{n}-2}$. The third is the most complicated one called aromatic hydrocarbons, also known 
as arenes. They always have at least one aromatic ring.

The main compound in cleaning gasoline are hydrocarbon, alcohols and ethers. The categories concluded Oxy-compound are listed as follows: alcohols like methyl, ethyl, TBA, and ethers like MTBE, TAME, ETBE, DME, DIPE, etc. Fuels with oxygen are enable to reduce the content of $\mathrm{CO}, \mathrm{HC}$ and NOX from exhausted fumes. However, these new fuels and technologies may have a negative influence on the environment by producing toxic components. Therefore, it is of great necessity for manufacturers to take action to solve this problem by detecting not only the exhausted components, but also these other potential emission from the vehicle.

This paper shows the methods to measure the concentration of gaseous hydrocarbon, to find and provide the product data of test equipment which have good performance of measurement. There are also something that have to be done, for example, how to reduce the proportion of alkenes and arenes.

\section{Species Types of Hydrocarbons}

\subsection{Ether}

Ether are one of a group of chemicals commonly known as oxygenates. MTBE is one of the ether in the fuel which can replace the tetraethyl lead and to increase its octane rating, helping prevent engine knocking [1]. It is a colorless and volatile liquid, which has stable chemical properties and help gasoline burn more completely. The car using fuel with MTBE will has stronger driving performance, meanwhile has lower tailpipe emission. However, it is also toxic. MTBE at very low concentration in groundwater will lead it undrinkable, soil will also be damaged by MTBE. Some other ethers additive have similar properties with MTBE.

\subsection{Alkane}

Alkane is an acyclic saturated hydrocarbon, and also the carbon atoms are arranged in a tree structure, and it has all the carbon-carbon bonds are single [2]. The number of carbon atoms seemed to be the size of the alkane. Alkanes are not active, so it can combine more active functional groups of biological molecules. And, actually, it can be used in commercial sources like petroleum and natural gas. Alkane can be divided into three groups: linear, branched and cyclic. The linear is like a snake structure while the branched is to separate the carbon backbone in one or more direction. The cyclic is linked to form a loop. The first two are called alkanes while the third one is cycloalkanes. Alkane which has more than three carbon atoms can be combined in various way to form structural isomers. The physical properties of alkanes are neither has color nor odor. The process of the measurement only included gaseous of alkane which means that there are only four types of alkanes: Methane $\left(\mathrm{CH}_{4}\right)$, Ethane $\left(\mathrm{C}_{2} \mathrm{H}_{6}\right)$, Propane $\left(\mathrm{C}_{3} \mathrm{H}_{8}\right)$ and Butane $\left(\mathrm{C}_{4} \mathrm{H}_{10}\right)$. Alkane cannot be degraded automatically in the environment when they released, they usually need some bacteria to metabolize the atom, and the one product is alcohol. Sometimes, the liquid alkane can be dangerous due to its highly flammable property. The risk is increasingly higher with the length of the carbon chain. The application of the alkane is up to how many carbon atoms they have, and propane is gas and can work as a fuel to vehicles that is worth to discuss in this paper. $\mathrm{S}$

\subsection{Alkene}

Alkene is an unsaturated hydrocarbon that contains at least one carbon-carbon bonds [2]. Alkene have two hydrogen atoms less than the alkane. The physical property of the alkene is similar to alkanes, which is combustible, odorless and colorless. The physical state is depend on molecular mass. Alkenes are stable compounds, but they are more reactive than Alkane types. It is the main part of the gasoline and has multiple usage. According to the new standard of many nations, alkene is considered to be polluted compounds in gasoline. In order to meet the new requirement, the alkene in gasoline must be transferred into isobutene and aromatic hydrocarbons.

\subsection{Alcohols}

As one of the clean and renewable energy source, alcohols are oxy-compound in which the hydroxyl functional group ($\mathrm{OH}$ ) is bound to a saturated carbon atom [2]. Some alcohols, mainly ethanol and methanol, can be made as mix fuel with gasoline. However, alcohols contain great toxicity. The toxicity of ethanol is largely caused by its primary metabolite, and other alcohols are substantially more poisonous than ethanol, partly because they take much longer to be metabolized and partly because their metabolism produces substances are even more toxic. If the effluent of alcohol fuel pollute river, it will be harmful to human body. Therefore, the concentration of alcohols in fuel should be controlled.

\subsection{Others}

The combustion products of gasoline are not only oxycarbide and oxynitride, but include $\mathrm{NMHC}$ (non-methane hydrocarbon) which are hydrocarbon $\left(\mathrm{C}_{2}-\mathrm{C}_{8}\right)$ that can be volatilized. The compound is still stable in gas situation in normal temperature status, and discharge to atmosphere. However, when the concentration of NHMC accumulates to a higher extend, they will be harmful to human directly and produce poisonous chemical smokes which extremely impair environment and human being. So, it is of great necessity to test the concentration of NHMC from exhausted fumes. The concentration of $\mathrm{THC}$ and $\mathrm{CH}_{4}$ can be tested by testing the concentration of NHMC.

\section{Check for Measurement Principles}

\subsection{Mass Spectrometry and Gas Chromatography}

Mass Spectrometry is a new approach in modern physics. The basic theory of this approach is to split the gaseous 
molecule into ions, and then separate the ions according to their mass. After that, the mass-to-charge ratio of ions and mass spectrum can be obtained by testing and recording system. The mass spectrum provides many significant information about molecule mass, included elements and the structure of molecule, which can analyze the isomers, the structure of organics and elements, and the variety of gaseous ions [3].

Gas chromatography is a chromatographic technique to separate and analyze the compound which are easily volatilize and not decomposed. The typical usage of gas chromatography is to test the fineness of certain compound and to separate the elements of mixture, meanwhile it can also test proportion of elements respectively. There is a long pipe with slit inside the gas chromatograph which is called chromatographic column. The different sample which have different chemical and physical property can move by various velocity from air because the samples have different interaction with stationary phase. When the compound floats from the end of the pipe, they can be detected by the equipment, and then form a signal which is transferred to electric signal to output. The stationary phase in chromatographic column is to separate different elements, and to make the elements floated from the end of column in different time periods. Other factors that influence the sequence of material floating from the column and the factors of maintained time include floating velocity and temperature, etc. Normally, the gaseous material which keep stable within $300^{\circ} \mathrm{C}$ can be tested quantificationally. However, gas chromatography cannot identify all parts of samples, and not all the samples can be detected by gas chromatography. The real thing to test by gas chromatography is a material can be separated from chromatographic column in certain time and can be tested by machine. The researchers should know that what kind of element in the samples and their concentration to make the result more persuasive. In the meanwhile, the material with low concentration cannot be shown in the chromatogram because of the separation of other materials with high concentration from the samples.

GC-MS has the best performance to solve this complicated problem, because velocitron is enable to identify relative molecule mass of all groups of elements. There are two main parts about GC-MS, the one is gas chromatography and the other is mass spectra. The gas chromatography have capillary column and the important factor is the size of the column which include length, width and thickness of liquid film and the other factor is the quality of stationary phase. When the samples float through the column, they can be separated by different chemical property. The molecule must be kept by column to make sure they are enable to float off the column in different time period, and then the molecule can be captured by velocitron. After that, the molecule is ionize, accelerated and deviated, finally, the velocitron can test the ionized molecule respectively. The usage of velocitron is to split every molecule into piece of ion and to test by their mass-to-charge ratio. Environmentally speaking, GC-MC has become increasingly popular to track continually contaminated organics, and the price of the equipment is dropped dramatically. To some extent, the reliability have been enhanced. Therefore, the GC-MS have a good advantage to use in the study of environment control.

\subsection{The Flame Ionization Detector}

The flame ionization detector (FID) is a cutting-edged equipment where the concentration of organic species can be tested in a gas stream. The FID with simple structure is increasingly popular to consider as a detector in gas chromatography. The volume of FID is normally under 1ul, and the response time is $1 \mathrm{~ms}$. It can be not only used in combination of packed column, but also can used with capillary column. The main property of FID is to response nearly every volatile compounds. Normally, the relative response to every hydrocarbon compounds is practically same, and so to the homolog with impurity atoms in hydrocarbon compounds. In addition, the temperature of FID cannot have an impact on properties, the sensitivity is almost the same from 80 to 200 degrees which is beneficial to quantify the compounds. However, the FID cannot test the permanent gases, water, $\mathrm{CO}, \mathrm{CO}_{2}, \mathrm{NXOY}$, and $\mathrm{H}_{2} \mathrm{~S}$.

\subsection{Infrared Spectroscopy}

In the organic molecule, the atoms constructing chemical bonds and functional groups are constantly in vibration situation. Therefore, if the infrared light are used to light the organic molecule, the chemical bonds and the functional groups of the molecule can absorb the vibration. Different types of chemical bonds and functional groups have different absorption frequency, so they are enable to be at different location on IR, according to this, the information of chemical bonds and functional groups in molecule can be obtained.

The strength and location of infrared absorption peak reveal the feature of molecule structure, which can help to identify the structure of unknown compound and make sure what the chemical genes are. The strength of infrared band spectrum is in alignment with the change of the dipole moment in molecule vibration, the higher the dipole moment changes, the more strength the band spectrum is. The change of the dipole moment is in relation to the inherent dipole moment of perssad, the more strength the pole of perssad is, the more changeable the dipole moment is, and so the absorption band. Then, the qualitative analysis and purity identification can be done. However, when the symmetry of molecule is at a higher level, the change of the dipole moment in vibration can be less, then the absorption band is weaker, so there is not all the vibration pattern can be captured by the IR.

The combination of the FTIR and dispersion spectrometer can enhance the precision of the test sharply. For example, the gas chromatography is enable to separate the compound of mixture, then connecting the FTIR to transfer the infrared spectroscopy. This technology and GC-MS are complementary. GC-IR measurement is extremely effective on identifying isomers. The main successful factor of the equipment is the interferograms are quickly to be captured, 
normally, less than one second.

\subsection{Raman Spectrum}

Raman scattering is an inflexible scattering which uses the energy of a laser and the range of the laser is around visible light, near-infrared light and near ultraviolet [4]. The laser is interactive with phonon system, which leads to increase or reduce the photon energy. According to this change of the energy, the pattern of the phonon can be founded. In other words, in the whole process of the Raman scattering, the interaction of the incident photon and the sample produce the scattered photon which has different wave length as the incident photons. It is similar to the basic principle of infrared absorbing spectrum, including qualitative analysis and quantitative analysis. The different thing is that the results of two technology are complimentary, because the symmetry molecule such as carbon dioxide which is nonpolar molecule and has little signal in IR has very strong signal in Raman scattering because of the high density of electron cloud, easy polarization. Raman spectrum has a variety of information which can be used in chemical identification, molecule structure, bond effectiveness, the environment of the sample and distribution of the inner stress.

Surface-Enhanced Raman Scattering (SERS). SERS is a phenomenon of unusual optical enhancement which happens in nano-scaled rough surface or particle system. Normally, the nano particle of gold and silver can be enhanced to as 1010 to 1011 as much of original Raman signal by laser which means the technique can test single molecules and identify the isomers. The spectra of most SERS experiments are similar to the non-surface enhanced spectra, the technology of SERS has all the advantages which the non-surface enhanced spectra have. In additional, more specific information of molecular structure can be present in the SERS spectrum which is not found in the traditional Raman spectrum, while other modes can disappear. The ability to analyze the construction of a nano-scaled mixture makes SERS substrates as a useful role in the field of environmental analysis, for instance, testing the polycyclic aromatics of exhausted fume from vehicles.

\section{Manufactures of Test Equipment}

\subsection{Horiba}

Horiba is a Japanese manufacturer of measuring and analytical instruments. They are good at producing equipment which can measure and analyze automobile exhaust fumes critically, and are environmentally-friendly. The company is increasingly popular among the measurement industry.

In 2005, Horiba branched the company to Germany by acquiring the German company Schenck Development Test Systems. Therefore they can expand the vehicles market product range from engine and driveline testing tools to brake testing and wind-tunnel balances. The
Horiba group now consists of about 42 companies, spread over about 15 countries, and it is one of the top 25 analytical and life sciences instrumentation companies in the world.

The researcher found that it is good to test the exhausted fumes from vehicles by the MEXA-7000 series provided by Horiba companies.

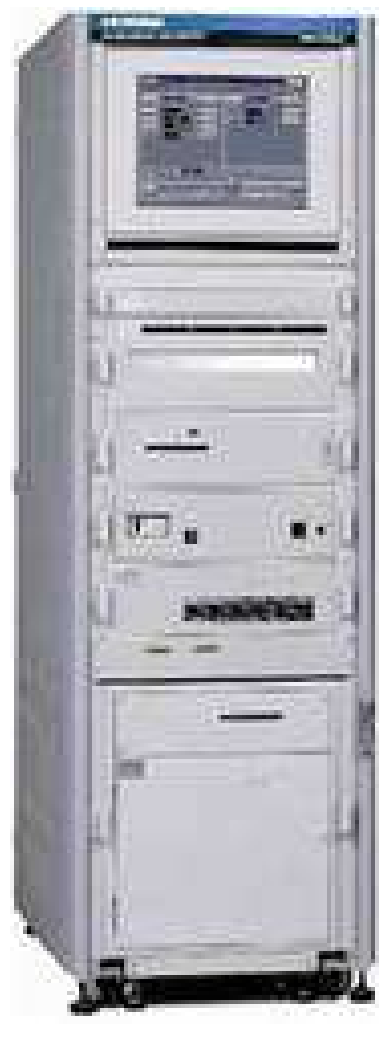

Figure 1. MEXA-7000 series.

The equipment includes up to 10 analyzers in a single, which can measure THC, $\mathrm{CO}, \mathrm{CO}_{2}, \mathrm{O}_{2}, \mathrm{NO} / \mathrm{NOx}, \mathrm{HC}$, $\mathrm{N}_{2} \mathrm{O}, \mathrm{SO}_{2}, \mathrm{CH}_{4}$, and EGR- $\mathrm{CO}_{2}$ over a wide dynamic range. It can measures emissions from gasoline, diesel, or alternative fuel engines. The accuracy of Super-low emission (SLE) analyzers for $\mathrm{HC}, \mathrm{NOx}$, and $\mathrm{CH}_{4}$ can down to $0.1 \mathrm{ppm}$.

\section{2. $A V L$}

AVL is an Austrian-based automotive consulting firm as well as an independent research institute. It is the extremely largest and privately owned company for the measurement of exhausted fumes from vehicles as well as analyzing the components in the fumes. And it is particularly useful since the THC, NMHC can be tested accurately.

The AVL SESAM i60 FT is a FTIR multi-component measurement system that measures numerous pre-calibrated gas components in a time-synchronous and dynamic way [5]. The concentration of all the hydrocarbons and $\mathrm{CH}_{4}$ can be tested accurately as well as calculating the density of gas with THC and NMHC. 

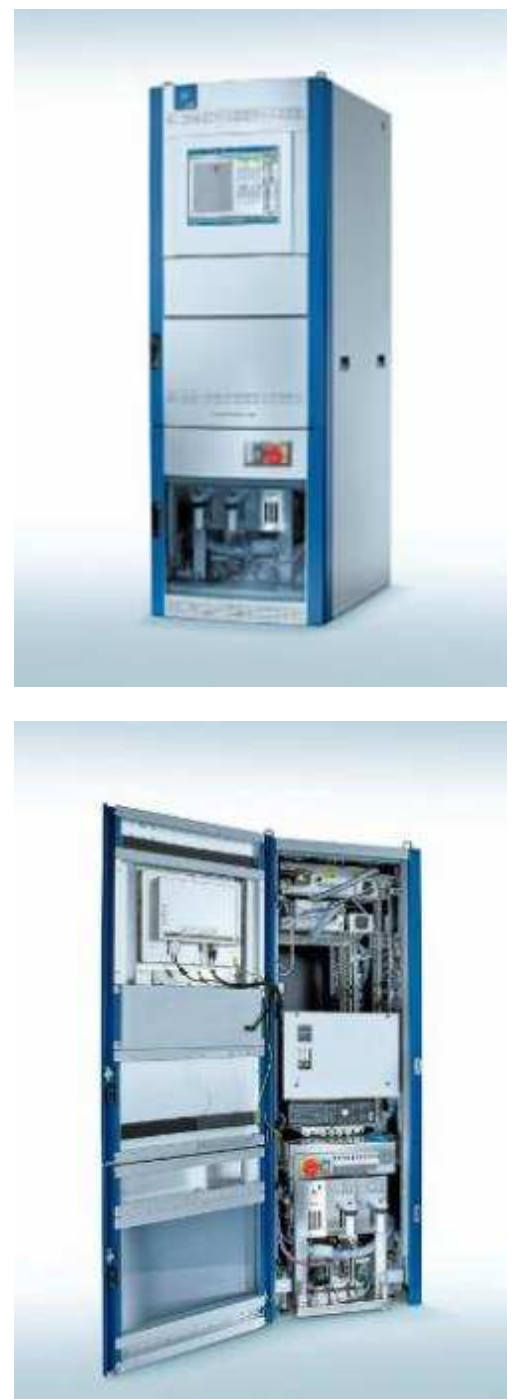

Figure 2. SESAM I60 FT.

\subsection{Ocean Optics}

Ocean Optics is an American company who contributed themselves to environmental protection, and they are increasingly popular among the manufacturers who produce the environmental testing equipment. The advantages of this firm is that they are enable to design new optical measurement technologies, active in every market all over the world.

Maya2000 Pro Spectrometers offer high sensitivity configurations that cover a range of 165-1000 nm [6]. Maya2000 Pro is an extra-sensitive and powerful spectrometers and they are flexibly adapted to many applications connection. The measurement techniques are variable: Absorbance, Fluorescence, Raman, Reflectance and transmittance.

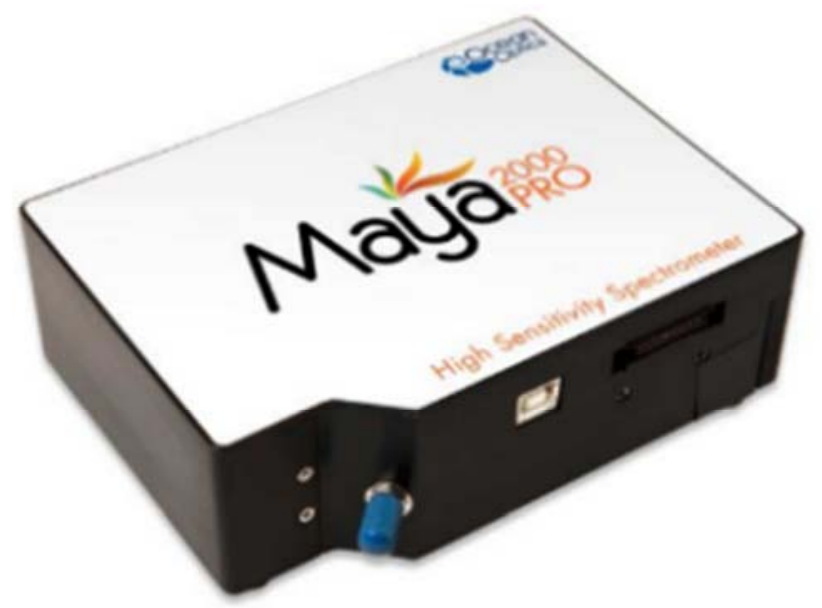

Figure 3. Maya2000 Pro.

Table 1. Statistics of Different Equipment.

\begin{tabular}{llll}
\hline & Horiba (MEXA-7000 series) & AVL (SESAM i60 FT) & Ocean Optics (Maya2000 Pro Spectrometers) \\
\hline Species type & THC & NMHC/THC & THC \\
principle & Heated FID & FTIR+FID & Raman \\
Precision\& accuracy & $0.1 \mathrm{ppm}$ & $0.5 \mathrm{~cm}-1$ & $780 \mathrm{~nm}$ \\
Temporal resolution & To t90 within $1.5 \mathrm{~s}$ & t 10 to 190 within $1 \mathrm{~s}$, fast response version & $6 \mathrm{~ms}$ to $5 \mathrm{~s}$ \\
Analyser temperature & $113^{\circ} \mathrm{C}$ or $191^{\circ} \mathrm{C}$ & within $300 \mathrm{~ms}$ & $0^{\circ} \mathrm{C}$ to $50^{\circ} \mathrm{C}$ \\
\hline
\end{tabular}

\section{Conclusion}

Because the exhaust gas is always within $200^{\circ} \mathrm{C}$ before it is emitted to the atmosphere, and the hydrocarbons are in gaseous form and volatile under high temperature, such as: NMHC. Meanwhile, the FID has good performance from 80 $-200^{\circ} \mathrm{C}$. So this paper account for the factors of temperature, species types, accuracy, etc., find three equipments from different manufactures (Horiba, AVL and Ocean Optics). By comparing this three types of equipment, the report found that the Horiba MEXA-7000 series have a good range of testing, the Maya 2000 Pro Spectrometers can get the highest precision with Raman, and the AVL SESAM i60 FT which use FTIR+FID can improve the testing temporal resolution. All of those equipments have advantages in different fields. At present, there is serious air pollution in the world, and with the increasing usage of cars, it is inevitable to decrease the emission standard of hydrocarbon. Thus, manufactures has to develop the equipment that has more accurate measurement methods, which is convenient for the vehicle manufactures to produce the environment friendly cars. 


\section{References}

[1] ZHONGXIAN, Zhang, "Environment and Green plants". Beijing: Tsinghua publication. PDF e-Book. 2005. pp. 85-88.

[2] International Union of Pure and Applied Chemistry, Org., "Compendium of Chemical Terminology". Gold Book. Oxford: Blackwell Science. PDF e-Book. 2014. pp. 53-60.

[3] SPARKMAN, O. David, "Spectrometry desk reference". Pittsburgh: Global View Publication. 2000.

[4] GARDINER, Derek J and Pierre R, GRAVES, "Practical Raman Spectroscopy", Introduction to Raman Scattering. Berlin: Springer-Verlag Berlin Heidelberg. PDF e-Book. 1989.

[5] AVL, "AVL SESAM i60 FT Multi Component Exhaust Measurement System”, Technical Data. Graz: AVL. 2017.

[6] OCEAN OPTICS, Maya 2000 Pro (Custom), "High Sensitivity Spectrometer”. United States: Ocean Optics. 1989.

[7] TÜV SÜD AUTOMOTIVE GMBH. "Exhaust emissions and fuel consumption measurements according to international regulations". Heimsheim: TÜV SÜD Automotive GMBH.

[8] AKEDA Kenji and Hideki KOIKE, "Motor Exhaust Gas Analyzer MEXA-7000 series". Downsizing and Modular Configuration of Analyzers. Japan: Horiba. 1995.

[9] AGILENT TECHNOLOGIES, "Fundamentals of GC Columns". In: homepage of Agilent Technologies. 19.11.2014.

[10] ENVIRONMENTAL PROTECTION AGENCY, "Conversion Factors for Hydrocarbon Emission Components". United States: Environment Protection Agency. Internet Documents. 2010.

[11] FRANK S, Weston, "Gas Chromatography-Infrared Spectroscopy" [PowerPoint-presentation]. GC-IR: Separate \& Identify. United States: Agilent Technology.

[12] HORIBA, "Emission Measurement Systems", MEXA-7000 Version 3. Japan: Horiba. 1996.

[13] CLARK, Jim, "Understanding Chemistry", The Mass Spectrometer. 2000. 